\title{
Quasi-distributed liquid level measurement with adaptable Optical Fiber transducers
}

\author{
M. Lomer, A. Quintela, MA Quintela, A. Cobo, J.M. López-Higuera \\ University of Cantabria, Photonics Engineering Group \\ Av. De los Castros s/n., 39005 Santander, Spain
}

\begin{abstract}
A level and flexible quasi-distributed liquid sensor based on the changes in the light transmittance in a plastic optical fiber cable is proposed. The measurement points are constituted by small areas created by side-polishing on a curved fiber and the removal of a portion of the core. These points are distributed and adapted on each full-turn of a spire of fiber built on a cylindrical tube vertically positioned in a tank. The changes between the refractive indexes of air and liquid generate a signal power proportional to the position and level of the liquid. The sensor system was successfully demonstrated in the laboratory and experimental results of three prototypes with 10, 8, and 5 measurement points and with bend radius of $5 \mathrm{~mm}$ are presented in this paper.
\end{abstract}

Keywords: Liquid level measurement, side-polishing, bend losses fiber, plastic optical fiber

\section{INTRODUCTION}

In many processes liquid level control plays a mayor role. A great variety of transducers and instruments based on electrical, acoustic, magnetic, and optical methods have been proposed for that measurement. The solutions using optical methods have the advantages of providing a high sensibility, immunity to electromagnetic interferences and security in explosive or aggressive atmospheres. In the last years, optical fiber sensors have contributed with different new solutions. When monomode and multimode glass fibers are used as part of the sensors, the resulting systems are well appreciated in metrology, especially those allowing a cost-effective implementation [1]. New findings in the fabrication of plastic optical fibers (POF) have contributed to expand their application fields; representing also a low cost solution [2-4]. In fact, the use of POF offers several advantages regarding their low weight, flexibility, easy handling, large core diameter and large numerical aperture. POF with diameters between 0.125 and $3 \mathrm{~mm}$, and typical transmission losses of $120 \mathrm{~dB} / \mathrm{Km}$ are already commercially available. Different configurations of liquid level sensors using optical fibers have been proposed in the last years. These are divided into sensors for punctual measurement [5-9] and sensors for continuous measurement [10-13]. All these methods need sophisticated optical arrays and additional optic components, in addition to the specific treatment of the signal to deduce the liquid level.

In this document, we present a quasi-distributed invasive liquid-level sensor based on the changes in light transmittance in a POF cable where the measurement points have been performed by side-polishing. The advantage of the proposed sensor in comparison to previous sensors is its easiness to be manufactured. The lateral polishing generates an elliptical surface where part of the core has been removed. The measurement points are laterally distributed along the fiber, placed at each loop of the $\mathrm{N}$ fiber full-turns. A change in the refraction index of the medium in contact with the core produces variations on the transmittance. These changes are detected in the output fiber ending. The lateral polishing produces moderated losses, allowing the measurement with several points along the entire fiber. In the following Sections a transducer implementation and experimental results obtained with the developed prototype are presented. Experimental results from three prototypes of 10, 8 and 5 measurement points performed on POF fiber spires with bend radius of $5 \mathrm{~mm}$ are also provided.

\section{TRANSDUCER CONSTRUCTION}

The construction of the quasi-distributed level sensor is obtained by means of a lateral polishing on the fiber spire according to the direction of the axis of the cylindrical tube. The array and the disposition of the three transducers within the tank are illustrated in Figure 1. Each full-turn of the fiber contains a measurement point and can be adapted to the

Third European Workshop on Optical Fibre Sensors, Antonello Cutolo, Brian Culshaw, José Miguel López-Higuera, Eds., Proceedings of SPIE Vol. 6619, 661917, (2007) $\cdot 0277-786 X / 07 / \$ 18 \cdot$ doi: 10.1117/12.738385 
height of the tank. Details of the design of the transducer have been developed in previous works by the authors [14]. The minimum range of level measurement between two points corresponds to the total diameter of the fiber. The spiral of the fiber is characterized by the bend radius of a full-turn spiral $R^{\prime}$, depending on the pitch $P$ and the fiber bend radius $R$, which is given by:

$$
\mathrm{R}^{\prime}=\mathrm{R}+(1 / \mathrm{R})[\mathrm{P} /(2 \pi)]^{2}
$$

and the fiber length per period

$$
L=\left[(2 \pi R)^{2}+P^{2}\right]^{1 / 2}
$$

Radius $R$ y $R^{\prime}$ are related to the fiber axis. $R^{\prime}$ and $L$ represent the distances for measurement and the fiber length required for the sensor in each measurement point.

The transducer I (Fig. 1) is constructed with a minimum period $(P=2.2 \mathrm{~mm})$ and allows to measure levels is the order of millimeters. For liquid tanks with higher heights the period of the fiber turn can be adapted to distances is the order of centimeters (transducer II). With the purpose of preserving the effect of the bend radius (5mm to $10 \mathrm{~mm}$ ), the value of $R^{\prime}$ does not have to exceed $10 \mathrm{~mm}$. As it is well-known, when the bend radius of a fiber increases, diminish the losses of propagation but sensitivity also diminishes by effect of the changes in air-liquid. For distances between level measurement points of several meters, the solutions consists of constructing full-turns containing the measurement point elaborated by polishing. This is illustrated by the configuration of transducer III.

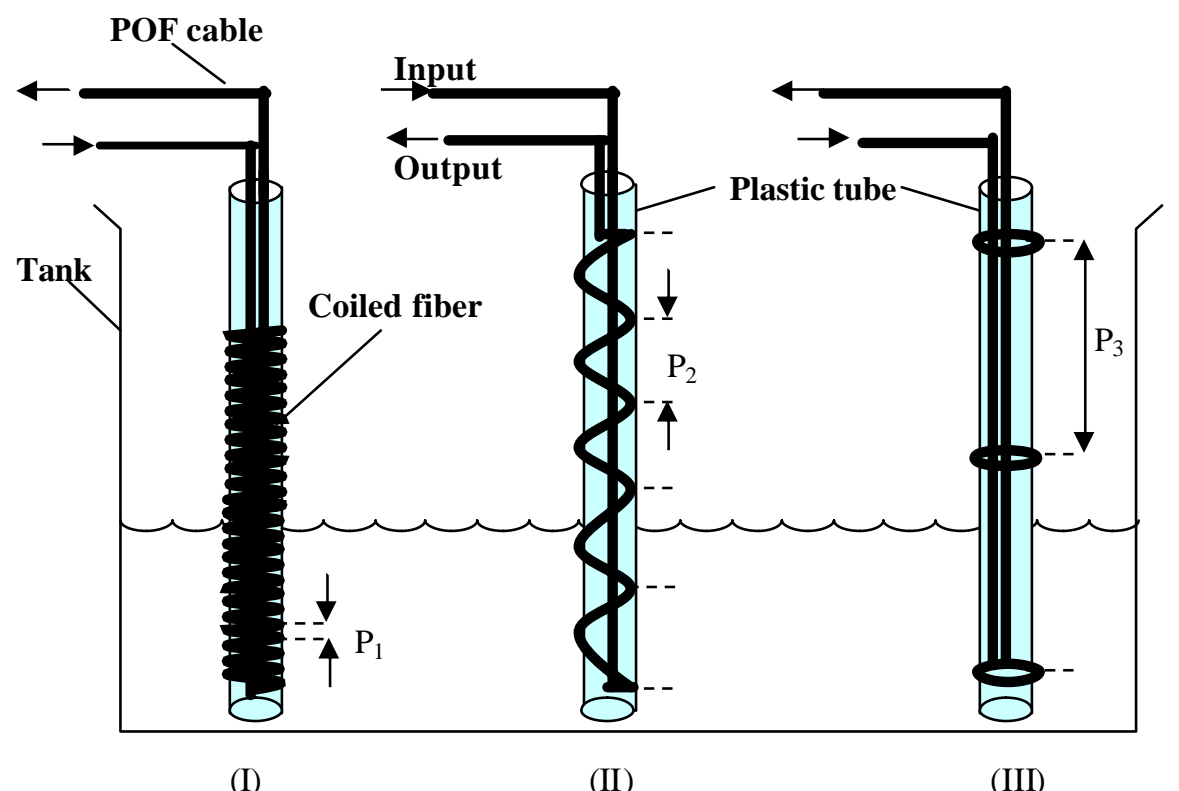

Figure 1 . Three different array of quasi-distributed liquid level sensor.

The construction of the level transducer is accomplished by complete curvatures of a cable POF on a cylindrical tube of radii $\rho$. The radii of curvature of the fiber is given by: $R=\rho+c$, where $\mathrm{c}$ is the radius of the cable POF (including the jacket); for a typical step-index PMMA-POF of $1 \mathrm{~mm}$ of diameter and a jacket thickness of $0.5 \mathrm{~mm}, \mathrm{c}$ has a value of $1 \mathrm{~mm}$. A hole is performed on an end of the cylindrical tube with the purpose of fixing the end of the fiber spire. This end is located at the bottom of the tank. Each complete turn of the fiber spire is successively constructed until completing the amount required for the level measurement. The two ends of the fiber are respectively derived toward the light source and the photo-detector. If the height of the container is several tens of millimetres, the distance between the points of level measurement (period $P$ ) corresponds to the minimum distance, i.e., the diameter of the cable POF. In the particular case of a tank of higher height, from the centimetres to several meters, the distance between the measurement points can be distributed in a periodic way by means of fibers curved with helix form. Images of the sensor set up and details are shown in Figure 2. 


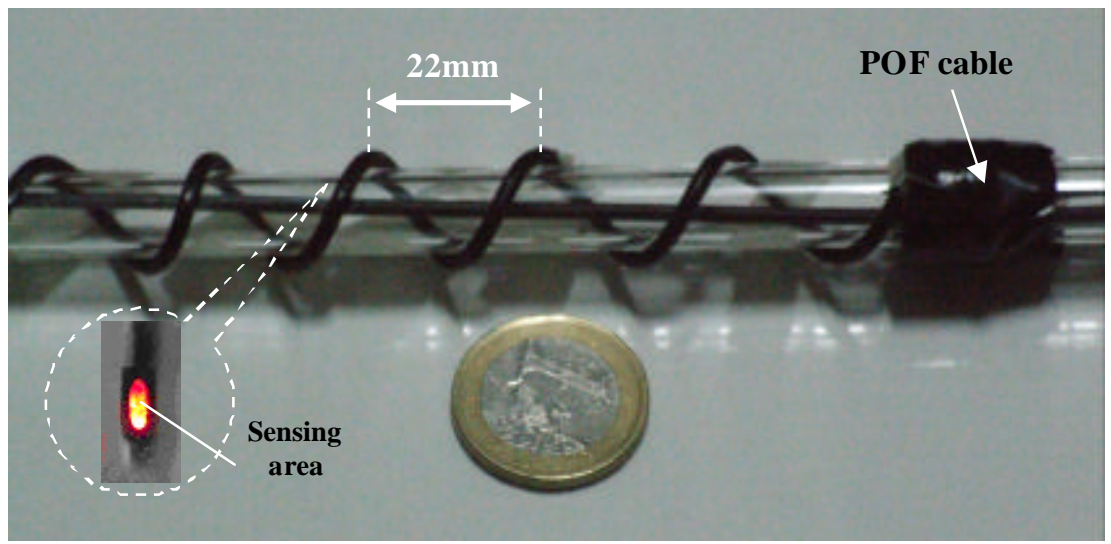

Figure 2. Transducer view with insets to illustrate the optical light radiation of one side polished loop.

\section{EXPERIMENTAL RESULTS}

Measurements of water level in a container have been performed with the proposed transducer (Fig. 1). The experimental results are obtained with three prototypes of transducers, with $R=5 \mathrm{~mm}$, and with a maximumpolished core thickness of $0.1 \mathrm{~mm}$, based on experimental results and trying to obtain a good sensitivity. A He -Ne laser $(\lambda=632.8 \mathrm{~nm})$ has been used as light source. Light is coupled into the fiber with a modulation frequency of $270 \mathrm{~Hz}$, which eliminates the background noise at the receiver. The signal from the sensor was detected using an optical power meter (Anritsu Corp.). In all the cases, the measurement of power of level " 0 " corresponds to the moment when the head transducers are in contact with the air. The first sensing point is located at the bottom of the water tank. The obtained experimental results in Figures 3 and 4 versus the number sensing points immersed in water are plotted.

Prototypes I and II have been of prepared to measure distances of some millimeters to several centimeters. The results show with clarity the changes in the losses by effect of the lateral polishing of the bend fiber identifing the position of the level of liquid in the tank. Fig. 3(a) shows the results of the level measurement with prototype I with 10 sensing points $(\mathrm{P}=2.2 \mathrm{~mm})$. The distance measured in a container has been $22 \mathrm{~mm}$. We observed that the changes in the losses by each full turn is $0.2 \mathrm{dBm}$, and $2.4 \mathrm{dBm}$ for the whole transducer. When the sensing distance increases, the losses also increase, but the sensitivity to the changes in the interface core-outside diminishes slightly, what can be observed in Figure 3(b). In this case the radius of the spire increases and modifies the curvature radius. The results of prototype III (Fig. 4.) show the measures performed on several meters of distance. In this case the changes in the transmittance of the light are evident. Each sensing point exhibits a difference $0.7 \mathrm{dBm}$ in the losses of attenuation.

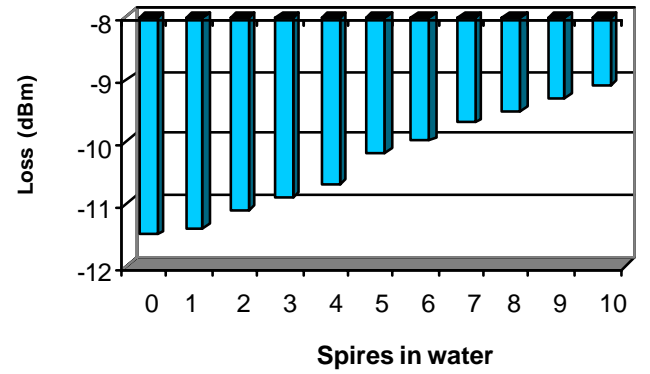

(a)

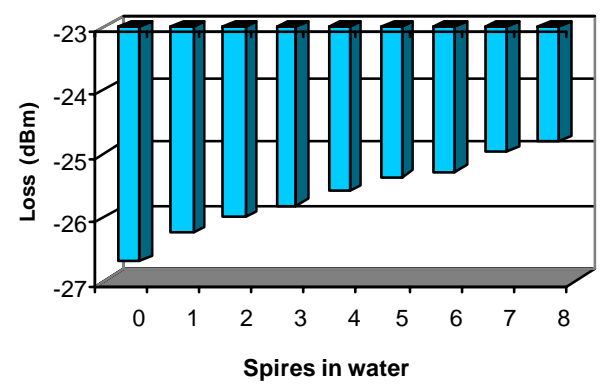

(b)

Figure 3. Experimental loss obtained as a function of distance for the transducer immersed in water,

a) 10 sensing points $(\mathrm{P}=2.2 \mathrm{~mm})$ and, b) 8 sensing points $(\mathrm{P}=22 \mathrm{~mm})$. 


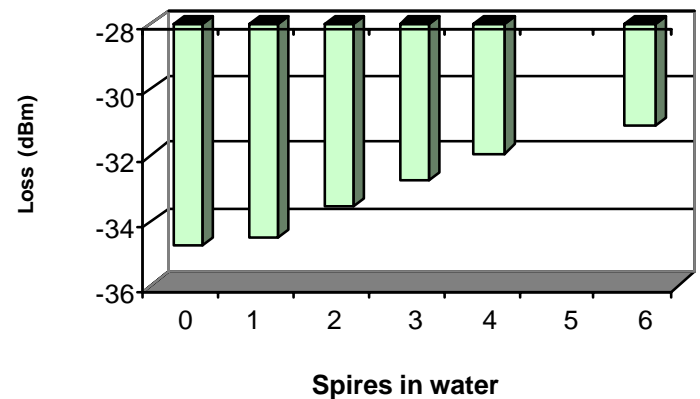

Fig. 4. Experimental loss obtained as a function of distance for a sensor immersed in water for 5 sensing points $(P=1 \mathrm{~m})$.

\section{CONCLUSIONS}

We have presented an adaptable quasi-distributed level sensor based on the extra attenuation that appears by bending and polishing plastic optical fibers. By polishing a section of the core on the bend the sensitivity of the device is improved. It has been demonstrated the feasibility of a quasi-distributed measurement system from some millimeters to several meters of distance. These range scales of level measurement can be integrated in a single fiber. The weak losses caused by polishing allows to have many sensing points. The precision of the measurement is given by the ellipse minor axis diameter formed by the initial polished $(2 \mathrm{x}=0.08 \mathrm{~mm})$. The advantages introduced by the use of POFs make it possible to develop very cost effective sensing systems.

\section{Acknowledgments}

The authors thank the Spanish Government Ministry of Science and Technology for its support the TEC2004-05936-C02-02 and TEC2005-08218-CO2 Projects.

\section{REFERENCES}

[1] J.M Lopez-Higuera Editor 2002 Handbook of Optical Fiber Sensing Technology (New York: Wiley).

[2] R.J. Bartlett, R.P. Chandy, P. Eldridge, D.F. Merchant, R. Morgan, and P.J. Scully "Plastic optical fiber sensor and devices", Trans Inst Measurement and Control, 22 431-457 (2000).

[3] M. Tekelloglu and B.D. Wood "Prediction of light-transmission losses in plastic optical fibers", Appl Opt 44 23182326 (2005)

[4] P. Polishuk P "Plastic optical fiber builds on MOST success", Laser Focus World 42 (March) 57-61 (2006)

[5] K. Spenner, et al. "Experimental investigation of fiber optic liquid level sensor and refractometers" Proc First Int Conf on Optical Fiber Sensor Conf(London 1983) pp 96-99 (1983)

[6] A. Wang, et al., "Fiber-optic Liquid-level sensor" Sensor and Actuators A 35 161-164 (1992)

[7] O. Raatikainen, I. Kassamakov, R. Kakanakov and M. Luukkala, "Fiber-optic Liquid-level sensor", Sensor and Actuators A, 58 93-97 (1997).

[8] M. Bottacini, et al., "All-plastic optical-fiber level sensor” Microwave Opt Technol Lett 46 520-522 (2005)

[9] V. Svirid, S. Khotiaintsev, P.L. Swart, "Linear and steplike characteristics in an optical fiber refractometric transducer with hemispherical detection element" Opt Eng 42 1383-1389 (2003).

[10] K. Iwamoto and I. Kamata, "Liquid-level sensor with optical fibers Appl Opt 31 51-54 (1992).

[11] C. Vazquez, A.B. Gonzalo, S. Vargas, J. Montalvo, "Multi-sensor system using plastic optical fibers for intrinsically safe level measurements" Sensor and Actuators A 116 22-32 (2004).

[12] F. Porez-Ocon, M. Rubino, J.M. Abril, P. Casanova, J.A. Martinez, "Fiber-optic liquid-level continuous gauge" Sensor and Actuators A 125 124-132 (2006).

[13] S. Khaliq, S.W. James and R. Tatam, 'Fiber-optic liquid-level sensor using a long-period grating", Opt Lett 26 1224-1226 (2001).

[14] M. Lomer et al., 'Low-Cost Quasi-Distributed Sensor Based on Bended Side-Polished Plastic Optical Fiber', in 18th Int. Optical Fiber Sensors Conference Technical, OFS-18 TuE-49 (2006). 